sensitive surveys at various wavelengths have reached deeper into distance and time, such counts have been mostly affected by the evolution of source populations with 'look-back' time. Evolution is thus a central element of cosmic history, and a prerequisite for the derivation of cosmic geometry ${ }^{9,10}$. Unfortunately, the deep-count data do not adequately constrain the evolution models, and conclusions based on models have been contradictory $^{10}$. In this context, the study of Windhorst et al. stands out for its achievement of solid progress simply based on meticulous observations that drive our best telescopes to new heights of performance.

George Helou is in the Infrared Processing and Analysis Center, California Institute of Technology, M/S 100-22, Pasadena, California 91125, USA.

\title{
Making selection work
}

\section{John F. Y. Brookfield}

A POTENTIALLY happy and fruitful courtship is taking place between what, until now, have largely been two distinct disciplines in biology. The disciplines are population genetics and that loose consortium of pursuits known as biotechnology. The common interest is the use of selection to optimize the performance of drugs, antibodies and enzymes, and it brought representatives of the two sides together at a meeting last month*.

Evolution by natural selection consists of a mutational process creating genetic alternatives, of which that with the highest fitness increases in frequency. Mutation, selection and inheritance thereby successively increase mean fitness. Despite its lack of foresight, in that it selects amongst variants only on the basis of their present performance, and not their potential for subsequent improvement, the power of the process can be seen in the phenomenal success of living things.

Correspondingly, in the design of many new processes and products, the most effective strategy has not been rational planning on the basis of knowledge, but rather the creation of a selective environment in which products are successively optimized on the basis of a 'forced' process of evolution. We need to know how best to carry out such a strategy, by asking, for example, what is the optimal number of variants to screen, how many we should select, how much mutation we should impose on them, and whether we should, by recombination, bring together different favoured variants in the same replicating molecule. All of these questions are closely analogous to those of traditional population genetics and of computer-based genetic algorithms, hence the rationale for the meeting.

Most simply, forced evolution starts with blind mutation followed by screening, as exemplified by recent progress in combinatorial chemistry. M. Pavia

*Biotechnology and Biological Sciences Research Council Workshop on Forced Evolution. Warwick, UK, 15-16 May 1995.

NATURE · VOL $375 \cdot 8$ JUNE 1995
(Sphinx Pharmaceuticals) described methods for producing a vast range of small organic molecules, each of which can be tested for a variety of biological activities. For amino-acid sequences, the astronomical number of possible permutations requires successive rounds of optimization and mutation. In this way, the capacity of the enzyme subtilisin to function in the organic solvent dimethylformamide has been greatly improved $(F$. Arnold, California Inst. of Technol.). The experimenter still has to test alternative proteins, however, and retain the genes encoding the best.

More rapid selection is possible if molecules can be automatically retained as a result of their activities. Ribozymes can be developed from initial pools of RNAs of random sequence through the use of selection in which only those variants capable of ligating themselves to an anchored RNA are used to form the next generation (D. Bartel, Whitehead Inst.). Ribozymes, as with the hypothesized enzymatic RNAs in the 'RNA world'1 at life's origin, carry their genotype with them, and selection of a molecule with enhanced activity also selects genetically. Evolution can only occur if the genotype is somehow coupled to the phenotype, as in early life where the move from an 'RNA world' to a 'ribonucleoprotein world' required the development of membranes so that only an improved RNA itself would receive the beneficial effects of the improved protein that it encoded. Libraries of antibodies displayed on phage (G. Winter, Laboratory of Molecular Biology, Cambridge) allow the coupling of protein phenotype and inheritance. A specific antibody displayed on the surface of the phage may bind its antigen; in this way, the attached phage, containing the genes encoding the antibody, is selected.

Notwithstanding the enormous power of forced evolution, participants at the meeting were reminded of the old story in which a tourist, seeking directions in an unfamiliar city, receives from a native the less-than-helpful advice: "Well, I wouldn't start from here". The point is that an initially successful variant, although better than its competitors, might be even further from the overall optimum than was the starting point of the process. A primary determinant of the efficacy of forced evolution is the presence or absence of such epistasis. Will an advantageous genotype get stuck on an 'adaptive peak'? On such a peak, all single mutations are detrimental, although a major improvement would be produced by the right set of multiple changes.

In Escherichia coli, replicate populations, all initially of the same clonal genotype, were subjected to a new glucose-based environment ${ }^{2,3}$. All replicates increased their fitness, but the phenotypes associated with this adaptation process differed. After 2,000 generations, the rate of improvement in fitness had dropped almost to nothing, despite continued mutation. This suggests that a range of possible mutations can adapt a genotype, but that, once some of these have occurred, subsequent mutations convey no advantage, despite their advantageousness in the genetic background of the initial clone. Genotypes thus evolve to different stable endpoints through the chance occurrence and fixation of different advantageous mutations ${ }^{4}$ - a phenomenon which, if general, would preclude the full explanation of organisms' phenotypes solely in terms of the selection imposed by their environments.

In applications of forced evolution, it seems that epistasis rarely traps molecules on adaptive peaks. Even if it does, strategies for escape are possible, such as recombination. A new method induces recombination in vitro through the use of primerless polymerase chain reactions with mixtures of randomly sheared molecules of different sequence ${ }^{5,6}$ (W. Stemmer, Affymax, Palo Alto). Again, close parallels are seen with population genetic explanations for the evolution of recombination.

Forced evolution will not be the solution to all problems in the design and optimization of new molecules and processes. But it may well be very helpful in tackling many of them, and in this the seemingly arcane field of theoretical population genetics may prove the most potent tool.

John F. Y. Brookfield is in the Department of Genetics, University of Nottingham, Queens Medical Centre, Nottingham NG7 2UH, UK.

1. Gilbert, W. Nature 319, 618 (1986).

2. Lenski, R. E. \& Travisano, M. Proc. natn. Acad. Sci. U.S.A. 91, 6808-6814 (1994)

3. Travisano, M. et al. Science 267, 87-90 (1995)

4. Mani, G. S. \& Clarke, B. C. Proc. R. Soc. B240, 29-37 (1990).

5. Stemmer, W.P. C. Nature 370, 389-391 (1994)

6. Stemmer, W. P. C. Proc. natn. Acad. Sci. U.S.A. 91 10747-10751(1994). 